\title{
Hot Topics in Surgical Management of Acute Diverticulitiss
}

\author{
Gian Andrea Binda ${ }^{1}$, Alfredo Papa ${ }^{2}$, Roberto Persiani ${ }^{3}$, Ricardo Escalante ${ }^{4}$, Enio Chavez de Oliveira ${ }^{5}$, Antonio Crucitti ${ }^{6}$, \\ Andrea Mazzari ${ }^{6}$, Alberto Biondi ${ }^{3}$, Savvas Papagrigoriadis ${ }^{7}$
}

1) Department of Surgery,

"Galliera" Hospital, Genoa, Italy

2) Division of

Internal Medicine and

Gastroenterology, CIC

"Columbus", Catholic

University, "A. Gemelli”

University Hospital, Rome,

Italy

3) Department of Surgical

Sciences, First General Surgery

Unit, Catholic University, „A.

Gemelli” University Hospital,

Rome, Italy

4) Universidad Central de

Venezuela, Loira Medical

Center, Caracas, Venezuela

5) Av, 470 - Hosp Ort de

Goiania (St Aeroporto),

Goiânia, Goiás (GO), Brazil

6) Division of General Surgery,

„Cristo Re” Hospital, Rome,

Italy

7) Department of Colorectal

Surgery, King‘s College

Hospital, London, United

Kingdom

\footnotetext{
Address for correspondence:

Gian Andrea Binda,MD

Department of Surgery,

"Galliera Hospital”, Genoa,

Italy

gianbinda1@gmail.com
}

\begin{abstract}
In this session different issues for the surgical management of diverticular disease DD) were considered. The first session debated about the antibiotic treatment for acute uncomplicated diverticulitis (AUD), and supports their use selectively rather than routinely in patients with AUD. The second session discussed the best surgical treatment for those patients. Open approach is a valid choice especially in acute setting, while the laparoscopic approach should be individualised according to the level of skills of the surgeon and the risk factors of the patient (such as obesity and state of health at the time of the operation). The third session debated about the peritoneal lavage and drainage, which is still a safe surgical procedure. However, it requires longer follow-up and results of other trials to draw an adequate conclusion.

The last session covers the current surgical certainties in managing complicated DD: 1 . urgent colectomy has higher mortality in immune-compromised patients, while in elective surgery is comparable with other populations; 2. laparoscopic peritoneal lavage (LPL) should be the choice in young/fit patients; 3. elective resection is safer in an inflammation free interval; 4. laparoscopic resection shows advantages in several outcomes (such as post-operative morbidity and lower stoma and re-operation rate); 5. in Hinchey III/fecal peritonitis, primary sigmoid resection and anastomosis (open or laparoscopic) could be proposed in young/ fit patient; 6 . in case of emergency surgery, Hartmann procedure (open or laparoscopic) must be considered in critically ill/unstable patient.
\end{abstract}

Key words: diverticular disease - acute diverticulitis - laparoscopic surgery - open surgery.

Abbreviations: AUD: acute uncomplicated diverticulitis; CRP: C-reactive protein; CT: computer tomography; HP: Hartmann procedure; IVF: intra-venous fluids; LPL: laparoscopic peritoneal lavage; PA: primary anastomosis; RCT: randomized controlled studies.

\section{ANTIBIOTIC TREATMENT FOR UNCOMPLICATED ACUTE DIVERTICULITIS}

\section{Evidence against}

AUD consists in acute inflammation of colonic diverticula confirmed by computer tomography without any of the following complications: free perforation, abscess, fistula or stricture [1]. Recently, the efficacy of antibiotics in the management of AUD has been questioned and some systematic reviews and meta-analysis have been published on the treatment of AUD without antibiotics [2-4]. Mocanu et al. [2] included in their meta-analysis a total of eight studies concluding that antibiotics use in patients with AUD was not associated with a reduction in major complications, readmissions, treatment failure, progression to complicated diverticulitis, or need for elective or emergent surgery, but increased the length of hospital stay. However, only two of the studies included in the metaanalyses were randomized controlled trials (RCTs), and the rest were observational studies [2-4]. The first RCT comparing antibiotic treatment with no antibiotics was the "AVOD" study including 623 patients [5]. Three hundered and nine patients were randomized to intravenous fluids (IVF) alone and 314 to antibiotics plus IVF [5]. No significant differences were found in complication rate $(p=0.302)$, need for surgery $(p=0.504)$ or length of stay ( $p=0.717)$ [5]. At the 12-months follow-up, recurrent diverticulitis rate was also similar in the two groups $(\mathrm{p}=0.881)$ [5]. The other RCT (DIABOLO trial) included 528 
patients: 262 who did not received antibiotics and 266 treated with antibiotics [6]. Also in this study no differences were found in complication rate $(\mathrm{p}=0.377)$, need for surgery $(\mathrm{p}=0.323)$, recurrence at 6 -months $(\mathrm{p}=0.494)$, time to recovery $(\mathrm{p}=0.151)$, morbidity rate $(p=0.221)$ or mortality rate $(p=0.432)[6]$. On the other hand, length of stay was significanly longer in the group treated with antibiotics $(p=0.006)[6]$. Other reasons against the use of antibiotics for AUD are: less adverse drug effects, in particular Clostridium difficile infection and dysbiosis (7); reduction of bacterial resistance [8] and decreased costs [9].

\section{Evidence pro}

Although conservative treatment of AUD without antibiotics has shown to be feasible, safe, and effective [4], there is some evidence and agreement that antibiotics are still indicated in immunocompromised patients, with comorbidities as chronic kidney disease, collagen-vascular disease, diabetes, severe signs of sepsis, in patients on chronic corticosteroid therapy, or pregnant females, as these patients have a higher risk of treatment failure $[4,10,11]$.

There is some evidence that high C-reactive protein (CRP) level (>170 mg/ml) [12] or initial computer tomography (CT) findings of fluid collection and longer inflamed colon [13] could be useful factors in selecting patients who could benefit from antibiotic treatment, but these preliminary data need to be confirmed by further studies.

The short follow-up which did not exceed 12 months, except in two studies, is also an important limitation as the recurrence rates of AUD represent only the short-term outcome whereas long-term recurrence was not investigated.

A potential increased risk of further elective surgery is a matter of concern [14]. In the Diabolo trial [15] within 2 years after a CT confirmed AUD, authors found a trend toward more elective surgery in the observation group (observation: $7.7 \%$ vs. antibiotic: $4.2 \%, \mathrm{p}=0.09$ ). The risk of elective sigmoid resection in the observation group could have been underestimated, as the study was not powered for this secondary outcome. If antibiotics reduce the risk of developing chronic sequel, then we should continue to treat AUD with antibiotics, especially if the alternative is an increased risk of surgery with its related morbidity.

Although many societies or guidelines advised that antibiotics should be used selectively rather than routinely in patients with AUD $[16,17]$, some international guidelines [18] still recommend the use of antibiotics in the management of AUD.

\section{OPEN OR LAPAROSCOPIC TREATMENT?}

\section{The open approach is better}

Optimal surgical treatment of acute diverticulitis (AD) remains poorly defined regarding patient selection, timing, and technical approach both in elective and urgent settings. Based on high quality evidence, laparoscopic resection with primary anastomosis (PA) is considered the preferred approach compared to elective colectomy provided that adequate expertise is available [18].

In emergency settings, the role and outcomes of different types of operations, PA with ostomy vs Hartmann procedure
(HP) and the approach, laparoscopic vs open, have not been well studied and data are limited to low quality RCTs, nonrandomized and retrospective studies.

Based on a common sense, general peritonitis in a hemodynamically unstable patient is a contraindication for $\mathrm{PA}$ and laparoscopic approach [19]. In these cases, laparotomy and HP are still the most commonly used procedures $[20,21]$.

The surgical treatment of acute complicated diverticulitis (ACD) in stable patients is still a matter of intense debate. Treatment goals in this setting are different: resolution of the sepsis and symptoms, shifting treatment from emergency to elective setting and maintaining intestinal continuity (22). To date, the aims of surgical RCTs on ACD were the comparison of the HP to PA with diverting loop ileostomy, PA without stoma, and laparoscopic lavage without resection. Each study used a different primary outcome, making it difficult to summarize or compare results [23].

Current guidelines and systematic reviews state that laparoscopy in the urgent setting should be restricted to selected cases in expert centers [23]. Unquestionably, advanced skills in minimally invasive emergency colorectal surgery are crucial for a successful laparoscopic treatment $[18,24]$. However, in the acute settings the presence of an experienced colorectal surgeon is not always realistic, and open surgery still represents a valid choice.

\section{The laparoscopic approach is safer}

Laparoscopic surgery has grown over the last decades, so has robotic surgery. The Sigma trial was a randomised trial which found that operating time was longer for laparoscopic surgery with a conversion rate of 19\%. However, there was less pain and less hospital stay with better quality of life at six weeks, but did not differ after six months [25]. A randomised trial by Gervaz et al. [26] in 2010 found no difference between open or laparoscopic sigmoidectomy for diverticulitis, except for a faster functional recovery and better cosmetic results in the laparoscopy group.

The DILALA RCT comparing laparoscopic lavage with bowel resection and colostomy (Hartmann's procedure) as treatment for perforated diverticulitis found that laparoscopic lavage was a better option for perforated diverticulitis with purulent peritonitis than open resection and colostomy [27].

Cirocchi et al. [28] published a systematic review and meta-analysis on comparison between laparoscopic and open surgery in diverticulitis. The meta-analysis suggested that elective laparoscopic surgery was a safe and appropriate option for patients with DD and was associated with lower overall morbidity $(\mathrm{p}=0.01)$ and minor complication rate $(\mathrm{p}=0.008)$.

Laparoscopic surgery has been shown to be feasible for diverticular fistulas. A study by Martinolich et al. [29] of 111 consecutive cases showed a high conversion rate of $34 \%$ but a significantly shorter stay of 5.8 versus 8.1 days in laparoscopy as opposed to open surgery.

The single incision approach is also found to be safe. A study by Galetin et al. in 2019 on 110 patients compared with 55 matched pairs of patients with either single-incision laparoscopic versus open sigmoidectomy for DD found that single-incision was safe and offered shorter hospital 
stay, decreased blood transfusions and decreased opioid requirements [30].

A study by Cassini et al. in 2018 compared robotic surgery to laparoscopic surgery on 156 consecutive patients with complicated diverticulitis and found that robotic surgery was equally safe compared with laparoscopic surgery and had lower morbidity ( $4.6 \%$ vs. $13 \%, \mathrm{p}=0.091)$ [31].

In conclusion, the available literature shows that laparoscopic surgery and even robotic surgery are feasible and safe in patients with diverticulitis, in elective and also in acute settings. However, the nature and methodology of the trials do not allow for a straightforward superiority conclusion. The level of skills of the surgeon and the risk factors of the patient such as obesity and state of health at the time of the operation determine the best approach for each patient and the surgical approach should be individualized according to those characteristics.

\section{PERITONEAL LAVAGE FOR PERFORATED DIVERTICULITIS}

\section{Evidence against}

DD is a common clinical condition in the Western world. Despite its silent evolution some patients, around 10 to $20 \%$, may present symptoms ranging from persistent pain to colon lifethreating conditions as colon perforation and peritonitis [32].

Treatment of perforated diverticulitis is old and at the beginning of the last century was based on laparotomy. In the 30 's the colostomy was added to the lavage without resection. Around 1950 resection and colostomy were the recommended approach. In 90's the resection and primary anastomosis were reported by some authors. In the last 20 years, laparoscopy and lavage for perforated diverticulitis were proposed to those patients with Hinchey III peritonitis [32, 33].

The laparoscopic approach has been demonstrated as a safe and effective treatment option in both elective and emergency settings of DD with all the advantages of the laparoscopic approach: fast recovery, less pain, shorter hospital stay and cost-effectiveness. However, large, prospective, randomized studies on LPL should be conducted to confirm these findings as a recommendation for purulent peritonitis Hinchey III. Hartmann's procedure is still acceptable, especially in highrisk patients [34].

Better clinical outcomes of patients submitted to LPL and sometimes with no need for further surgical treatment of the DD has been reported by some authors. However, the data published so far is controversial [23, 35]: no high-quality data were found to support the laparoscopic approach; highquality studies showed that LPL was associated with increased morbidity.

To date, three RCTs on LPL have been published and results are conflicting and inconsistent [34]. These facts warrant further research and debate.

\section{Evidence pro}

Over the years, morbidity and mortality during emergency surgery for complicated diverticulitis has been significant. Many alternatives have been adopted to improve these statistics and the safety of patients.
In 1996 O'Sullivan et al. [36, 37] proposed inspection of the colon and laparoscopic peritoneal lavage (LPL) for patients with peritonitis without gross fecal contamination [36, 37].

Cirocchi et al. [38], in a systematic review including papers from January 1992 to February 2012, reviewed multiple parameters in 19 articles, consisting of 10 cohort studies, 8 case series, and 1 controlled clinical trial. They concluded that LPL should be considered an effective and safe option for the treatment of patients with sigmoid diverticulitis with Hinchey stage III peritonitis; it can also be considered a "bridge" surgical step combined with a delayed and elective laparoscopic sigmoidectomy to avoid a Hartmann procedure.

Regenbogen et al. [39] conducted a systemic review of the recent primary reports on the decision-making, technical aspects, and outcomes of surgery for acute, recurrent, and chronic sigmoid diverticulitis. They found that complicated recurrence after recovery from an uncomplicated episode of diverticulitis was rare $(<5 \%)$ and that more frequent recurrence did not increase the risk for complications.

It seems that in this century, with the best resources, best technology, advanced laparoscopic techniques, and widestream antibiotics, we are going back to the technique that was initially proposed by Rolleston [40] more than 100 years ago. Some papers report that LPL is a safe, superior and effective alternative of surgical treatment for perforated diverticulitis, Hinchey III $[41,42]$. Other papers don't support this technique [43].

In a short series, Escalante et al. [44[ reported good results for laparoscopic lavage and suggested that a strict selection of patients, without comorbidities is necessary.

In conclusion, peritoneal lavage and drainage is a safe surgical procedure. However, longer follow-up and results of other trials will be necessary to draw an adequate conclusion.

\section{SURGICAL CERTAINTIES ON DIVERTICULAR DISEASE}

DD is common in industrialized countries, having a complicated course in $10-20 \%$ of the cases. After a first attack, $20-30 \%$ of patients undergo surgery, $50 \%$ of them in emergency. Among population $15-40 \%$ tend to be young (less than 50ys) with a growing percentage of acute presentation (9.5\%) [16]. Mortality is between 10-20\% in emergency operations, and 2\% in elective resections National Inpatient Sample Database in USA reported 314.000 admittance per years and 50.000 bowel resections, annually.

\section{Immunocompromised patients}

Immunocompromised patients (kidney failure, organ transplant, corticosteroids, HIV and CD $4<200$ cell/microL), usually have a more severe first episode of $\mathrm{AD}$ and a major risk of emergency surgical treatment. After the success of the medical treatment, immunocompromised patients should be addressed for elective surgery like other patients [45].

\section{Laparoscopic lavage}

First described in 1996, LPL is a conservative treatment in selected Hinchey II / IIIpatients, as well as in I or II, after the failure of medical treatment. Success or failure of LPL depends 
on the experience of the operator, the correct intraoperative detection of different grades of Hinchey diverticulitis, a complete and careful washout, abdominal drains in Douglas' and Morrisson's pouch. LPL can avoid stoma creation in up to $38 \%$ to $51 \%$ of cases; the 12 -month stoma-free rate is an important patient-centered outcome. Today, LPL for Hinchey III should be performed predominantly in clinical studies $[46,47]$.

\section{Reconstruction after sigmoid colectomy}

Perforated left-sided diverticulitis with generalized peritonitis (Hinchey III/IV) is a life-threatening clinical situation which occurs frequently in the emergency or surgical departments; sigmoid colectomy with primary resection and anastomosis (PRA) or HP are considered. Only $7.6 \%$ of the patients get a primary anastomosis. The results of RCTs showed a similar morbidity and mortality in short term follow-up and reduced length of stay in HP patients; the long term followup showed more definitive stomas, a worse quality of life and higher morbidity after stoma reversal. The meta-analysis of RCTs [48] showed no statistical differences in mortality or major morbidity between PRA and HP. Stoma reversal rate was higher in PRA compared to HP, and morbidity was lower in PRA patients. Laparoscopic emergency sigmoid colectomy offers a small advantage in complications and length of stay; general peritonitis is often still regarded as a contraindication for the laparoscopic approach, especially in case of fecal peritonitis. Damage control surgery must be suggested for clinically unstable, old/fragile patients with diverticular peritonitis and severe septic shock. The defunctionalized loop has a role in some cases like a not prepared colon, intraoperative findings, high risk patients (corticosteroids, age, comorbidity, local situation), technical reasons (surgical device) or quality of residual colonic tissue.

\section{CONCLUSION}

Surgical certainties in complicated DD are difficult to assess. In immunocompromised patients, emergency colectomy is associated with higher mortality, in elective setting comparable mortality and major morbidity are associated. LPL should be an operative choice in young/fit patients also in Hinchey III patients; further investigational studies are needed. Elective resection is safer in an inflammation free interval. Laparoscopic resection shows advantages in terms of per operatory morbidity, length of stay, lower stoma and re-operation rate, decrease of abdominal wall complications, but needs advanced technical skills and referring centers. In Hinchey III/fecal peritonitis sigmoid resection and PRA (open or laparoscopic) could be proposed in young/fit patient; in case of emergency surgery, HP (open or laparoscopic) must be considered in critically ill/unstable patient (damage control) [49].

\section{REFERENCES}

1. Tursi A, Papa A, Danese S. Review article: the pathophysiology and medical management of diverticulosis and diverticular disease of the colon. Aliment Pharmacol Ther 2015;42:664-684. doi:10.1111/apt.13322
2. Mocanu V, Dang JT, Switzer N, et al. The role of antibiotics in acute uncomplicated diverticulitis: A systematic review and meta-analysis. Am J Surg 2018;216:604-609. doi:10.1016/j.amjsurg.2018.01.039

3. Desai M, Fathallah J, Natulapati V, Saligram S. Antibiotics versus no antibiotics for acute uncomplicated diverticulitis: a systematic review and meta-analysis. Dis Colon Rectum 2019;62:1005-1012. doi:10.1097/ DCR.0000000000001324

4. Emile SH, Elfeki H, Sakr A, Shalaby M. Management of acute uncomplicated diverticulitis without antibiotics:a systematic review, meta-analysis, and meta-regression of predictors of treatment failure. Tech Coloproctol 2018;22:499-509. doi:10.1007/s10151-018-1817-y

5. Chabok A, Pahlman L, Hjern F, Haapaniemi S, Smedh K; AVOD Study Group. Randomized clinical trial of antibiotics in acute uncomplicated diverticulitis. Br J Surg 2012;99:532-539. doi:10.1002/bjs.8688

6. Daniels L, Ünlü Ç, de Korte N, et al; Dutch Diverticular Disease (3D) Collaborative Study Group. Randomized clinical trial of observational versus antibiotic treatment for a first episode of CTproven uncomplicated acute diverticulitis. Br J Surg 2017;104:52-61. doi:10.1002/bjs.10309

7. Tamma PD, Avdic E, Li DX, Dzintars K, Cosgrove SE. Association of Adverse Events With Antibiotic Use in Hospitalized Patients. JAMA Intern Med 2017;177:1308-1315. doi:10.1001/ jamainternmed.2017.1938

8. Alvarez-Uria G, Gandra S, Mandal S, Laxminarayan R. Global forecast of antimicrobial resistance in invasive isolates of Escherichia coli and Klebsiella pneumoniae. Int J Infect Dis 2018;68:50-53. doi:10.1016/j. ijid.2018.01.011

9. Denny KJ, Gartside JG, Alcorn K, Cross JW, Maloney S, Keijzers G Appropriateness of antibiotic prescribing in the emergency department. J Antimicrob Chemother 2019;74:515-520. doi:10.1093/jac/dky447

10. Rezapour M, Stollman N. Antibiotics in Uncomplicated Acute Diverticulitis: to give or not to give? Inflamm Intest Dis 2018;3:75-79. doi:10.1159/000489631

11. Jaung R, Kularatna M, Robertson JP, et al. Uncomplicated acute diverticulitis: identifying risk factors for severe outcomes. World J Surg 2017;41:2258-2265. doi:10.1007/s00268-017-4012-9

12. Bolkenstein HE, Draaisma WA, van de Wall B, Consten E, Broeders I. Treatment of acute uncomplicated diverticulitis without antibiotics:risk factors for treatment failure. Int J Colorectal Dis 2018;33:863-869. doi:10.1007/s00384-018-3055-1

13. Van Dijk ST, Daniels L, Nio CY, et al. Predictive factors on CT imaging for progression of uncomplicated into complicated acute diverticulitis. Int J Colorectal Dis 2017;32:1693-1698. doi:10.1007/s00384-017-29190

14. Peery AF. It's Actually a Little Complicated:Antibiotics for Uncomplicated Diverticulitis. Am J Gastroenterol 2018;113:949-950. doi:10.1038/ s41395-018-0159-8

15. Van Dijk ST, Daniels L, Unlu C, et al; Dutch Diverticular Disease (3D) Collaborative Study Group. Long-Term Effects of Omitting Antibiotics in Uncomplicated Acute Diverticulitis. Am J Gastroenterol 2018;113:1045-1052. doi:10.1038/s41395-018-0030-y

16. Binda GA, Cuomo R, Laghi A, et al; Italian Society of Colon and Rectal Surgery. Practice parameters for the treatment of colonic diverticular disease:Italian Society of Colon and Rectal Surgery (SICCR) guidelines. Tech Coloproctol 2015;19:615-626. doi:10.1007/s10151-015-1370-x

17. Stollman N, Smalley W, Hirano I; AGA Institute Clinical Guidelines Committee. American Gastroenterological Association Institute guideline on the management of acute diverticulitis. Gastroenterology 2015;149:1944-1949. doi:10.1053/j.gastro.2015.10.003 
18. Feingold D, Steele SR, Lee S, et al. Practice parameters for the treatment of sigmoid diverticulitis. Dis Colon Rectum 2014;57:284-294. doi:10.1097/DCR.0000000000000075

19. Strate LL, Morris AM. Epidemiology, Pathophysiology, and Treatment of Diverticulitis. Gastroenterology 2019;156:1282-1298.e1. doi:10.1053/j. gastro.2018.12.033

20. Sartelli M, Catena F, Ansaloni L, et al. WSES Guidelines for the management of acute left sided colonic diverticulitis in the emergency setting. World J Emerg Surg 2016;11:37. doi:10.1186/s13017-016-0095-0

21. Vennix S, Boersema GS, Buskens CJ, et al. Emergency Laparoscopic Sigmoidectomy for Perforated Diverticulitis with Generalised Peritonitis: A Systematic Review. Dig Surg 2016;33:1-7. doi:10.1159/000441150

22. Morris AM, Kin C. Surgery for diverticulitis in the 21st century: recent evidence. Minerva Gastroenterol Dietol 2017;63:158-162. doi:10.23736/ S1121-421X.17.02389-3

23. Beyer-Berjot L, Maggiori L, Loiseau D et al. Emergency Surgery in Acute Diverticulitis:A Systematic Review. Dis Colon Rectum 2019 Jan 25. doi:10.1097/DCR.0000000000001327

24. Di Saverio S. Emergency laparoscopy: a new emerging discipline for treating abdominal emergencies attempting to minimize costs and invasiveness and maximize outcomes and patients' comfort. J Trauma Acute Care Surg 2014;77:338-350. doi:10.1097/TA.0000000000000288

25. Klarenbeek BR, Veenhof AA, Bergamaschi R, et al. Laparoscopic sigmoid resection for diverticulitis decreases major morbidity rates: a randomized control trial: short-term results of the Sigma Trial. Ann Surg 2009;249:39-44. doi:10.1097/SLA.0b013e31818e416a

26. Gervaz P, Inan I, Perneger T, Schiffer E, Morel P. A prospective, randomized, single-blind comparison of laparoscopic versus open sigmoid colectomy for diverticulitis. Ann Surg 2010;252:3-8. doi:10.1097/SLA.0b013e3181dbb5a5

27. Kohl A, Rosenberg J, Bock D, et al. Two-year results of the randomized clinical trial DILALA comparing laparoscopic lavage with resection as treatment for perforated diverticulitis. Br J Surg 2018;105:1128-1134. doi:10.1002/bjs.10839

28. Cirocchi R, Farinella E, Trastulli S, Sciannameo F, Audisio RA. Elective sigmoid colectomy for diverticular disease. Laparoscopic vs open surgery: a systematic review. Colorectal Dis 2012;14:671-683. doi:10.1111/j.1463-1318.2011.02666.x

29. Martinolich J, Croasdale DR, Bhakta AS, et al. Laparoscopic Surgery for Diverticular Fistulas: Outcomes of 111 Consecutive Cases at a Single Institution. J Gastrointest Surg 2019;23:1015-1021. doi:10.1007/s11605018-3950-3

30. Galetin A, Rink AD, Vestweber B, Vestweber KH, Galetin T. Single-Incision Laparoscopic versus Open Sigmoidectomy for Diverticular Disease:A Disease-Stratified Matched-Pair Analysis. Dig Surg 2019;28:1-9. doi:10.1159/000497449

31. Cassini D, Depalma N, Grieco M, Cirocchi R, Manoochehri F, Baldazzi G. Robotic pelvic dissection as surgical treatment of complicated diverticulitis in elective settings: a comparative study with fully laparoscopic procedure. Surg Endosc 2019;33:2583-2590. doi:10.1007/ s00464-018-6553-x

32. Vermeulen J, Lange JF. Treatment of Perforated Diverticulitis with Generalized Peritonitis:Past, Present, and Future. World J Surg 2010;34:587-593. doi:10.1007/s00268-009-0372-0

33. Cirocchi R, Afshar S, Di Saverio S, et al. A historical review of surgery for peritonitis secondary to acute colonic diverticulitis: from Lockhart-
Mummery to evidence-based medicine. World J Emerg Surg 2017;12:14. doi:10.1186/s13017-017-0120-y

34. Marshall JR, Buchwald PL, Gandhi J, et al. Laparoscopic Lavage in the Management of Hinchey Grade III Diverticulitis: A Systematic Review. Ann Surg 2017;265:670-676. doi:10.1097/SLA.0000000000002005

35. Slim K, Le Roy B. Laparoscopic peritoneal lavage for perforated sigmoid diverticulitis - an example of surgical research failure. Colorectal Dis 2017;19:208. doi: 10.1111/codi.13598

36. O'Sullivan GC, Murphy D, O’Brien MG, Ireland A. Laparoscopic management of generalized peritonitis due to perforated colonic diverticula. Am J Surg 1996;171:432-434. doi:10.1016/S00029610(97)89625-0

37. Rogers AC, Collins D, O’Sullivan GC, Winter DC. Laparoscopic lavage for perforated diverticulitis: a population analysis. Dis Colon Rectum 2012;55:932-938. doi:10.1097/DCR.0b013e31826178d0

38. Cirocchi R, Trastulli S, Vettoretto N, et al. Laparoscopic peritoneal lavage: a definitive treatment for diverticular peritonitis or a "bridge" to elective laparoscopic sigmoidectomy?: a systematic review. Medicine (Baltimore) 2015;94:e334. doi:10.1097/MD.0000000000000334

39. Regenbogen SE, Hardiman KM, Hendren S, Morris AM. Surgery for diverticulitis in the 21st century: a systematic review. JAMA Surg 2014;149:292-303. doi:10.1001/jamasurg.2013.5477

40. Rolleston HD. Pericolitis sinistra. Lancet 1905;854:860

41. Gralista P, Moris D, Vailas M, et al. Laparoscopic Approach in Colonic Diverticulitis: Dispelling Myths and Misperceptions. Surg Laparosc Endosc Percutan Tech 2017;27:73-82. doi:10.1097/ SLE.0000000000000386

42. Angenete E, Bock D, Rosenberg J, Haglind E. Laparoscopic lavage is superior to colon resection for perforated purulent diverticulitis-a meta-analysis. Int J Colorectal Dis 2017;32:163-169. doi:10.1007/ s00384-016-2636-0

43. Sneiders D, Lambrichts D, Swank H, et al. Long-term follow-up of a multicentre cohort study on laparoscopic peritoneal lavage for perforated diverticulitis. Colorectal Dis 2019;21:705-714. doi:10.1111/ codi.14586

44. Escalante R, Bustamante-Lopez L, Lizcano A, Acosta MB. Peritoneal Lavage in Complicated Acute Diverticulitis: Back to the Future. J Clin Gastroenterol 2016;50 Suppl 1:S83-S85. doi:10.1097/ MCG.0000000000000619

45. Al-Khamis A, Abou Khalil J, Demian M, et al. Sigmoid Colectomy for Acute Diverticulitis in Immunosuppressed vs Immunocompetent Patients: Outcomes From the ACS-NSQIP Database. Dis Colon Rectum 2016;59:101-109. doi:10.1097/DCR.0000000000000513

46. Acuna SA, Wood T, Chesney TR, et al. Operative Strategies for Perforated Diverticulitis: A Systematic Review and Meta-analysis. Dis Colon Rectum 2018;61:1442-1453. doi:10.1097/DCR.0000000000001149

47. Binda GA, Bonino MA, Siri G, et al; LLO Study Group. Multicentre international trial of laparoscopic lavage for Hinchey III acute diverticulitis (LLO Study). Br J Surg 2018;105:1835-1843. doi:10.1002/bjs.10916

48. Schmidt S, Ismail T, Puhan MA, Soll C, Breitenstein S. Meta-analysis of surgical strategies in perforated left colonic diverticulitis with generalized peritonitis. Langenbecks Arch Surg 2018;403:425-433. doi 10.1007/s00423-018-1686-x

49. Lambrichts DPV, Birindelli A, Tonini V, et al. The Multidisciplinary Management of Acute Complicated Diverticulitis. Inflamm Intest Dis 2018;3:80-90. doi:10.1159/000486677 